\title{
2018 Canadian Consortium on Neurodegeneration in Aging (CCNA) Science Day Abstracts (Montréal, Québec - October 1-2, 2018)
}

http://dx.doi.org/10.5770/cgj.21.320

\section{Masters Students}

Whole Brain Volume Relative to Decade of Birth in Three Cohorts: Alzheimer's Disease, Mild Cognitive Impairment, and Cognitively Normal

M. Amin Banihashemi ${ }^{1,2}$, Shraddha Sapkota ${ }^{1}$, Susan E. Bronskill ${ }^{2,3}$, Donald T. Stuss ${ }^{2,4}$, Sandra E. Black ${ }^{1,2}$. ${ }^{1}$ Sunnybrook Health Sciences Center, ${ }^{2}$ University of Toronto, ${ }^{3}$ Institute for Clinical Evaluative Sciences, ${ }^{4}$ Baycrest Centre.

Background: Age-specific risk and incidence of dementia appears to be decreasing internationally. A current hypothesis for this decrease is that societal investments over time have led to improvements in population health with resultant effects on cognition and brain reserve. To assess if brain reserve has indeed been increasing amongst individuals born in later decades of the 20th century, we examined whole brain volume as an indicator of global neurodegeneration from the $1920 \mathrm{~s}$ to the $1950 \mathrm{~s}$.

Methods: Participants (at baseline visit: $\mathrm{n}=1,580$, age $=73.84 \pm 7.23$ years, \%female $=43.8 \%$ ) from three diagnostic groups (cognitively normal individuals [CN] $(\mathrm{n}=407)$, Mild Cognitive Impairment $[\mathrm{MCI}](\mathrm{n}=852)$, and Alzheimer's disease $[\mathrm{AD}](\mathrm{n}=321))$ were included from the Alzheimer's Disease Neuroimaging Initiative (ADNI). The relationship between whole brain volume (expressed as percentage of intracranial volume) and decade of birth was assessed with a linear regression model in $\mathrm{R}$ (version 3.4.3) accounting for age, sex, years of education, Apolipoprotein- $\varepsilon 4$ alleles, and Mini-Mental State Exam.

Results: Participants born in later decades had significantly greater percentage whole brain volumes in all three diagnostic groups [CN: $\beta=3.96$ (95\% CI: 3.00-4.93), $p<0.001$, adj. $\mathrm{R}^{2}=0.39$; MCI: $\beta=4.16$ (3.38-4.94), $p<.001$, adj. $\mathrm{R}^{2}=.37$; $\mathrm{AD}: \beta=4.66$ (3.57-5.74), $p<.001$, adj. $\left.\mathrm{R}^{2}=.26\right)$ ]. Adjusted $\mathrm{R}^{2}$ values were low suggesting low model predictive ability.

Conclusions: Participants born in later decades show a trend towards higher brain reserve as indexed by brain volume. This suggests a possible contribution to decline in age-specific incidence of dementia. We plan to examine this finding further in the COMPASS-ND cohort and improve data exploration and modeling.

\section{Analyzing Mobility Patterns of Older Adults with Dementia Using GPS Technology}

Sayeh Bayat ${ }^{1}$, Gary Naglie ${ }^{2}$, Mark Rapoport ${ }^{3}$, Bing Ye ${ }^{4}$, Elaine Stasiulis ${ }^{2}$, and Alex Mihailidis ${ }^{1}$ on behalf of the CCNA Driving Cessation Team Co-Investigators and CCNA study group. ${ }^{1}$ University of Toronto, ${ }^{2}$ Baycrest Health Sciences, ${ }^{3}$ Sunnybrook Research Institute, ${ }^{4}$ Toronto Rehabilitation Institute.

Background: Persons with dementia (PWD) must eventually stop driving, which poses challenges to maintaining their mobility. Out-of-home mobility is frequently measured in terms of life-space, defined as the spatial area through which a person moves. Life-space is traditionally selfreported using questionnaires or travel diaries and is thus subject to inaccuracies.

Objectives: To develop and validate GPS-based life-space measures, assess the feasibility of using GPS technology to measure different dimensions of mobility, and compare mobility patterns between PWD and controls.

Method: In the first of this two-phase pilot trial, two dyads of PWD and their caregivers and two controls carried the GPS device when traveling outside their homes for 4 weeks. Spatial and temporal features of mobility, including area, perimeter, and frequency of trips, were measured from the GPS trajectories. Participants' transportation modes were also detected.

Preliminary Results: Moderate agreements were observed between algorithms and travel diaries for number and duration of trips. The PWD demonstrated smaller life-space compared to control participants, including smaller travel 
area $(134.93 \pm 17.02$ vs. $201.29 \pm 27.22 \mathrm{~km} 2)$, perimeter (50.92 \pm 7.47 vs. $62.86 \pm 2.48 \mathrm{~km})$, and frequency of daily trips away from home $(1.15 \pm 0.15 \mathrm{vs} .1 .85 \pm 0.05 \mathrm{~km})$. The PWD also showed a preference for car commutes over other transportation forms.

Implications: The new GPS-based life-space construct shows much promise in objectively and accurately measuring life-space, which is valuable for longterm monitoring of older adults' out-of-home mobility behaviours. Next steps involve validation of algorithms with a larger sample of participants.

\section{Characterizing Neuropathology and Cholinergic Dysfunction in the Rostral Prefrontal Cortex}

Selena P. Maxwell, Meghan K. Cash, Dr. Sultan Darvesh. Dalhousie University.

Background: Tauopathies are a group of neurodegenerative disorders (NDDs) characterized by abnormal deposition of the microtubule-associated protein tau. Brodmann Area 10 (BA10), located at the most rostral part of the prefrontal cortex, contributes to many aspects of cognition including working memory, and executive function. Acetylcholine is a neurotransmitter produced by choline acetyltransferase (ChAT) containing neurons and regulated by the enzymes acetylcholinesterase (AChE) and butyrylcholinesterase (BChE). Cholinergic neurotransmission is integral to maintaining working memory and executive function. Impairment of working memory and executive function are shared clinical features of Alzheimer's disease (AD), corticobasal degeneration (CBD), frontotemporal dementia (FTD), and progressive supranuclear palsy (PSP).

Purpose: The aim of this study is to characterize neuropathological and cholinergic alterations in BA10 of $\mathrm{AD}, \mathrm{CBD}, \mathrm{FTD}$, and PSP brains.

Methods: Immunohistochemical techniques will be used to examine the distributions of $\beta$-amyloid, tau and ChAT. Histochemical techniques will be used to examine $\mathrm{AChE}$, and $\mathrm{BChE}$ activity. Tandem mass tag proteomics will be used to quantify changes in cholinergic synaptic proteins. Sex- and age-matched brain tissues from control, AD, FTD, PSP, and CBD brain tissues will be compared.

Results: The results of this study will further our understanding of differential BA10 pathology in NDDs including identifying the role of $\mathrm{BChE}$ in cholinergic dysfunction. Preliminary results indicate BChE does not associate with amyloid plaques or tau tangles in PSP in addition to FTD and CBD as previously described.
Implications: Examination of BA10 will facilitate understanding the mechanistic underpinnings of deficits in working memory and executive function in tauopathy.

Using Case Management (CM) to Address the Needs of Dementia and High Frailty Patients: Perceptions of Healthcare Professionals

Matthew Hacker Teper ${ }^{1}$, Catherine Hudon ${ }^{2}$, Isabelle Vedel ${ }^{1}$. ${ }^{1}$ McGill University, ${ }^{2}$ Université de Sherbrooke.

Context/Objectives: Case management (CM) is a collaborative process assessment, planning, facilitation, care coordination, evaluation and advocacy for patients. Despite established benefits of CM for the care of elderly and chronically ill patients, implementation of CM remains challenging in Québec. The research objectives are (1) to understand how healthcare professionals view the structural transition towards collaborative health interventions like $\mathrm{CM}$; (2) to highlight potential barriers, successes and limitations to establishing and sustaining CM usage according to Family Medicine Group (FMG) professionals; and (3) by extension, to identify strategies that will lead to better sustained CM into FMGs.

Methods: We are conducting a multi-centered qualitative case study in FMGs across Québec. Cases will be purposively selected to represent maximum variability in terms of the degree to which $\mathrm{CM}$ has been implemented into FMGs and the clientele that FMGs service (Alzheimer's patients vs. high frailty users). Clinicians within each FMG will be engaged with focus groups, semi-structured interviews, and participant observation. Document analysis will also be performed. Thematic analysis with be conducted as data sources are triangulated.

Results/Anticipated Results: Data collection is currently being conducted, and preliminary results will be available for the presentation. This research is intended to identify professional perceptions of $\mathrm{CM}$, and to outline strategies for improving the uptake and sustainability of $\mathrm{CM}$ in FMGs. This may result in improved care for dementia and elderly patients.

Conclusions: Our qualitative case study will use the perceptions of FMG healthcare professionals to understand how to improve CM function and sustainability in FMGs.

Case Management of Culturally Diverse Dementia Patients and Caregivers: A Mixed Methods Study in a Montreal Family Medicine Group 
Xin Qiang Yang ${ }^{1}$, Vladimir Khanassov ${ }^{1,2}$, Isabelle Vedel ${ }^{1}$. ${ }^{1}$ McGill University, ${ }^{2}$ Herzl Family Practice Centre, Jewish General Hospital.

Context: Dementia is a set of neurodegenerative diseases characterized by cognitive decline, causing important morbidity and mortality. Pending definitive treatments, clinicians look to better utilize the available resources to improve patients' and their caregivers' quality of life. The Quebec Alzheimer Plan has implemented case managers in family medicine groups, often nurses or social workers, who empower patients in their decision-making and coordinate their care, collaborating with the broader healthcare team. However, little evidence exists on how this model of care navigates culturally sensitive contexts, especially given the local ethnic landscape of Quebec.

Objectives: We will determine the impact of case management across culturally diverse groups of dementia patients and their caregivers, by quantifying their met and unmet needs. We will also describe the subjective experiences of these different groups.

Methods: The investigations will be part of a larger study within the Canadian Consortium on Neurodegeneration and Aging, examining dementia patients' and caregivers' needs and their use of healthcare services. For the current project, we aim to recruit 50 pairs of patients and caregivers living in the community. First, we will perform a cross-sectional study using validated questionnaires to investigate the study participants' needs. We will determine in what ways these results differ across cultural groups. Second, we will perform a qualitative descriptive study, exploring the study participants' experiences. We will identify recurring themes, contributing factors, current interventions and new solutions.

Conclusions: The study will investigate whether the cultural diversity of dementia patients and caregivers is accommodated for in the context of family medicine groups. We hope to inform future programs and policies to better care for all members of this vulnerable population and further improve their quality of life.

\section{MD, MD/PhD, or PhD Students}

Is There Evidence to Support the Use of Art Therapy as an Effective Modality to Reduce Neuropsychiatric Symptoms of Dementia? A Preliminary Report

Elena Guseva ${ }^{1,2}$, Ovidiu Lungu ${ }^{2,3,4}$, Isabelle Vedel ${ }^{1}$, Sondra Goldman ${ }^{5,2}$, Machelle Wilchesky ${ }^{1,2,6}$. ${ }^{1}$ McGill University, Department of Family Medicine, ${ }^{2}$ Donald Berman Maimonides Geriatric Centre, ${ }^{3}$ University of Montreal,
${ }^{4}$ Centre de recherche de l'institut universitaire de gériatrie de Montreal, ${ }^{5}$ International Art Hives network, Concordia University, ${ }^{6}$ Lady Davis Institute for Medical Research, Jewish General Hospital.

Background: Art therapy prioritizes non-verbal communication, providing a unique opportunity for sensory stimulation, social interaction, and creativity for persons with dementia faced with declining cognitive and verbal abilities. As such, art therapy has increasingly been used in this population in healthcare and other settings. Despite an emphasis on non-pharmacological approaches to symptomatic management as a response to the ever-growing dementia epidemic, the effectiveness of this powerful therapeutic modality in a diverse dementia population has yet to be established.

Objectives: To evaluate clinical application of art therapy interventions tailored to improve psychological and behavioral symptoms associated with advanced stages of dementia.

Methods: Studies pertaining specifically to the effects art therapy on dementia symptoms were extracted from the work of Cowl and Gaugler (2014) that reviewed 112 studies related to the gamut of Creative Arts Therapies (including art, music and drama therapy) in treatment of dementia from inception until November 2012.

Results: A total of 9 studies satisfied our study criteria that reported on 15 patient outcomes that included agitation, apathy, behavior, cognition, depression, and social engagement.

Discussion: An insignificant number of case studies and small trials reflect the need for more rigorous scientific enquiry in order to establish methods, assess effectiveness, and define optimal conditions for the use of art therapy in dementia.

Implications: A comprehensive systematic review evaluating the evidence pertaining to of the effectiveness of art therapy interventions to improve psychological and behavioral symptoms of dementia from inception until 2018 is planned.

Can Adapted Art Therapy Interventions Reduce Neuropsychiatric Symptoms in Long-Term Care Residents with Moderate to Advanced Alzheimer's Disease (AD)?

Elena Guseva ${ }^{1,2}$, Ovidiu Lungu ${ }^{2,3,4}$, Isabelle Vedel ${ }^{1}$, Sondra Goldman $^{5,2}$, Machelle Wilchesky1,2,6. ${ }^{1}$ McGill University, Department of Family Medicine, ${ }^{2}$ Donald Berman Maimonides Geriatric Centre, ${ }^{3}$ University of Montreal, 
${ }^{4}$ Centre de recherche de l'institut universitaire de gériatrie de Montreal, ${ }^{5}$ International Art Hives network, Concordia University, ${ }^{6}$ Lady Davis Institute for Medical Research, Jewish General Hospital.

Background: The risks, limited effectiveness, and resource constraints associated with clinical management strategies for neuropsychiatric symptoms (NPS) in dementia have led to an increased focus on art therapy (AT) as a possible non-pharmacological option. AD-related progressive visuospatial and perceptual deficits, however, restrict the range and effectiveness of AT interventions that can be employed. Adapting AT interventions to account for ADrelated visual pathology may prove beneficial at reducing NPS in this population; yet this hypothesis has not been investigated to date.

Objectives: To 1) Provide proof-of-concept evidence that visually-enhanced $\mathrm{AD}$-adapted $\mathrm{AT}$ may be more effective than non-adapted AT interventions at reducing two NPS (agitation/aggression and apathy); and 2) Evaluate the feasibility and utility of wearable devices to assess NPS changes in this population.

Methods: A pilot mixed-methods crossover study will be conducted to compare visually enhanced AD-adapted and non-adapted AT interventions. Qualitative data obtained from video recorded AT sessions will be integrated with quantitative physiological data from wearable devices both during and between AT sessions.

Expected Results: We anticipate providing preliminary evidence that visually-enhanced, AD-adapted AT is effective at reducing NPS in this population.

Implications of the research: This project will be the first dementia study to examine the effectiveness of AT interventions by collecting and analyzing objective neurophysiological measurements using wearable devices. A protocol for a larger clinical trial will be developed using the data obtained from this pilot in order to rigorously evaluate this study question.

\section{A Highly Predictive Signature of Cognition and Brain Atrophy for Progression to Alzheimer's Dementia}

Angela Tam ${ }^{1,2,3}$, Christian Dansereau ${ }^{1,4}$, Yasser ItturiaMedina $^{3}$, Sebastian Urchs ${ }^{3}$, Pierre Orban ${ }^{1,5,6}$, John Breitner ${ }^{2,3}$, Pierre Bellec ${ }^{1,4}$. ${ }^{1}$ Centre de Recherche de l'Institut Universitaire de Gériatrie de Montréal, ${ }^{2}$ Douglas Hospital Research Centre, McGill University, ${ }^{3}$ McGill University, ${ }^{4}$ Université de Montréal, ${ }^{5}$ Centre de Recherche de l'Institut Universitaire en Santé Mentale de Montréal, ${ }^{6}$ Département de Psychiatrie, Université de Montréal.

Abstract: Patients with mild cognitive impairment (MCI) are at risk of progressing to Alzheimer's dementia, yet only a fraction of them do. We explore here whether a very high-risk MCI subgroup can be identified using additional cognitive assessments and structural neuroimaging. A multimodal signature of Alzheimer's dementia was first extracted using machine learning tools in the ADNI1 sample, and was comprised of cognitive deficits across multiple domains as well as atrophy in temporal, parietal and occipital regions. We then validated the predictive value of this signature on two MCI cohorts. In ADNI1 ( $\mathrm{N}=235$ ), the presence of the signature predicted progression to dementia over three years with $80.4 \%$ positive predictive value, adjusted for a "typical" MCI baseline rate of 33\% (95.6\% specificity, $55.1 \%$ sensitivity). These results were replicated in ADNI2 ( $\mathrm{N}=235$ ), with $87.8 \%$ adjusted positive predictive value (96.7\% specificity, $47.3 \%$ sensitivity). Our results demonstrate that, even for widely used markers, marked improvement in positive predictive value over the literature can be achieved by focusing on a subgroup of individuals with similar brain characteristics. The signature can be readily applied for the enrichment of clinical trials.

\section{Crowd Sourced Quality Control of Brain Registration on the Zooniverse Platform}

Yassine Benhajali ${ }^{1,2}$, Helen Spiers ${ }^{3}$, AmanPreet Badhwar ${ }^{3,4}$, Sebastian Urchs ${ }^{5}$, Laura Trouille ${ }^{6}$, Pierre Bellec ${ }^{2,4}$. ${ }^{1}$ Anthropology, University of Montreal, Canada, ${ }^{2}$ CRIUGM, University of Montreal, Canada, ${ }^{3}$ Department of Physics, University of Oxford, UK, ${ }^{4}$ DIRO, University of Montreal, Canada, ${ }^{5} \mathrm{MNI}$, McGill University, Canada, ${ }^{6}$ Adler Planetarium, Chicago, USA.

Introduction: Despite widespread usage, there is no validated quality control (QC) procedure for MRI brain registration. In this work, we assess the reliability of a QC protocol in raters with prior experience (PE), and compare these raters with a consensus vote derived from hundreds of raters with no prior experience in registration QC (NE), recruited through the zooniverse crowdsourcing Internet platform [1].

Methods: A QC procedure was developed through user feedback over the past 5 years, and includes a series of key anatomical landmarks and associated confidence intervals [2] for 3 ratings: OK, Maybe or Fail. Based on ratings generated by YB and PB, we selected $100 \mathrm{~T} 1$ images from the ADHD $(\mathrm{n}=86)$ and COBRE $(\mathrm{n}=14)$ databases with $25 \mathrm{OK}, 51$ Maybe and 24 Fail. Images were then rated by $240 \mathrm{NE}$ and $4 \mathrm{PE}$ raters on Zooniverse. Each PE raters rated all 100 images. 
We selected $44 \mathrm{NE}$ raters who rated a minimum of $15 \mathrm{~T} 1$ images. NE and PE raters were included to generate two consensus (maximal vote) ratings. We used the kappa metric to test agreement between PE raters as well as the NE and PE consensus across all three levels of rating (OK, Maybe, Fail).

Results \& Conclusions: The results showed good agreement (Kappa mean: 0.68, std: 17) within PE raters, as well as between the NE and PE consensus. NE and PE consensus reached a kappa of 0.68 . Crowdsourcing of QC for brain registration is thus a promising avenue to establish reliable ratings on large data, which will be necessary to train automated machine learning QC algorithms.

1. Zooniverse at https://www.zooniverse.org/projects/ simexp/brain-match

2. Yassine, B. \& Pierre, B. figshare (2016).doi:10.6084/ m9.figshare.4204845.v1

\section{Comparing a Neuropathological Index with Traditional Pathology in Predicting Alzheimer's Dementia}

Lindsay Wallace ${ }^{1}$, Olga Theou ${ }^{1}$, Judith Godin ${ }^{1}$, Kenneth Rockwood $^{1,2}$, Melissa Andrew ${ }^{1,2}$. ${ }^{1}$ Dalhousie University, ${ }^{2}$ Centre for Health Care of the Elderly, Nova Scotia Health Authority.

Background: The hallmark neuropathological features of Alzheimer's disease (AD) don't correlate well with clinical dementia, suggesting cognitive impairment may be multifactorial in older adults with AD. We aimed to assess whether an index of diverse neuropathological features was more strongly associated with Alzheimer's-type dementia than traditional AD neuropathological hallmarks.

Methods: This was a cross-sectional analysis of data from the Rush Memory and Aging Project. We constructed a neuropathology index (NPI) using the deficit accumulation approach, as the mean of 10 variables coded between 0 (no pathology) and 1 (severe pathology): percentage of amyloid $\beta$, neurofibrillary tangle density, presence of Lewy bodies, hippocampal sclerosis, cerebral infarcts, cerebral amyloid angiopathy, arteriolosclerosis, atherosclerosis, and TDP43. A traditional pathology score included plaques (diffuse/ neuritic) and tangles. A 41-item frailty index of clinical health data was also calculated for each individual. Cognitive status was determined as $\mathrm{AD}$ or no dementia by clinical consensus (all other forms of dementia were excluded).

Results: The mean age of 645 included participants was $89.7 \pm 6.2$ years, $68 \%$ female. The NPI ranged from $0-0.87$, mean $0.36 \pm 0.16$. In a logistic regression model controlling for age, sex, and frailty, both NPI and traditional pathology were significantly associated with dementia diagnosis $(p<0.001)$. The NPI outperformed the traditional pathology measure in its ability to classify dementia status (C-statistic $0.80,95 \%$ CI $0.77-0.85$ vs. $0.74,0.70-0.78$ ).

Conclusion: An NPI captures information over and above traditional hallmark pathological measures of $\mathrm{AD}$ and may help characterize the multifactorial etiologic pathway of dementia in AD.

\section{Indigenous Older Adults Requiring Dementia Care: Making Space for Technology}

Danette Starblanket ${ }^{1}$, Carrie Bourassa ${ }^{2}$. ${ }^{1}$ University of Saskatchewan, ${ }^{2}$ First Nations University of Canada.

Background: Accessibility and readiness are key areas that require attention and strategic approaches in order to incorporate technologies and improve the current quality of life for Indigenous Older Adults Requiring Dementia Care.

Research Aim/question(s): To identify technology use amongst Indigenous older adults living with dementia and how they can be aligned with technologies using Indigenous community-based research methodologies.

Methods: We partnered with Indigenous older adults, caregivers, health practitioners and a Community Research Advisory Committee in the File Hills Qu'Appelle Tribal Council in southern Saskatchewan. Using a combination of Indigenous research methods and community-based involvement, interview participants were recruited through a purposive sampling procedure based on their firsthand knowledge of dementia programs and services and their own experiences. Interview data was gathered in focus groups and individual interviews. Qualitative data was collected from caregivers of older adults requiring dementia care, health care providers and older adults living with dementia.

Outcomes: Examined the barriers to access caused by infrastructure and cultural appropriateness. Addressed the various barriers that impacteffective technological development (e.g. access to technology and services, connectivity issues, unique culture). Explored the utility of technological services in dementia care for Indigenous communities.

Conclusion: Indigenous older adults requiring dementia care and residing in rural areas require specific technology in order to improve their quality of life and in order to age in place. 
Describing the Trends in Primary Care Continuity and Acute Hospital Use in Quebec from 2000 to 2015 for Community-Dwelling Persons with Dementia

Claire Godard-Sebillotte ${ }^{1}$, Nadia Sourial ${ }^{1,2}$, Louis Rochette ${ }^{3}$, Marine Hardouin ${ }^{2}$, Eric Pelletier ${ }^{3}$, Erin Strumpf ${ }^{1}$, Isabelle Vedel ${ }^{1,2} .{ }^{1}$ McGill University, ${ }^{2}$ Lady Davis Institute, Jewish General Hospital, ${ }^{3}$ Institut national de santé publique (INSPQ).

Context: Persons with dementia (PWD) have twice the acute hospital use as older persons without dementia, which adds strain to overburdened healthcare systems, impacts patients' and caregivers' quality of life and is associated with increased risk of adverse outcomes. Reducing avoidable hospitalizations in PWD is thus a global healthcare priority. While primary care continuity could be an important determinant, there is, to date, no description of the trends over time in primary care continuity and acute hospital use in PWD. Our objective is to describe those trends in Quebec.

Methods: Design: Repeated, cross-sectional 1-year cohort studies (2000-2015). Population: community-dwelling 65+ persons with incident dementia in Quebec. Outcomes: Primary care continuity (Bice-Boxerman index two-year before diagnosis), rate of Emergency Department (ED) visits or hospital admissions. Data source: Linkage of five Quebec provincial health administrative databases. Analysis: Description of trends over time, with age and sex standardized rates.

Results: Between 2000 and 2015, the number of incident cases increased (9217 to 15961). Visits to primary care ( 15/ year) were stable over time while cognition specialist visits slightly increased (3/year to 4/year). Primary care continuity remained constant (average Bice-Boxerman index $=0.316$ (99\%CI 0.315-0.318)). Rate of hospitalization decreased ( $44 \%$ to $40 \%$ ), rate of ED visit remained stable ( $60 \%)$.

Conclusions: This study is the first description of primary care continuity and acute hospital use over time in PWD. The relationship between both variables in PWD is essential and needs to be further explored, to inform healthcare policies aiming at reducing avoidable acute hospital use in this population.

Cerebrocholesterol and Tumor Necrosis Factor: Markers of Agitation Severity and Treatment Response to Nabilone in Patients with Moderate-To-Severe Alzheimer's Disease

Myuri Ruthirakuhan ${ }^{1,2}$, Nathan Herrmann ${ }^{1,3}$, Ana C. Andreazza $^{2}$, Damien Gallagher ${ }^{3}$, Nicolaas Paul L.G.
Verhoeff $^{4}$, Sandra E. Black ${ }^{1}$, Krista L. Lanctôt ${ }^{1,2,3}$. ${ }^{1}$ Hurvitz Brain Sciences Program, Sunnybrook Research Institute, ${ }^{2}$ University of Toronto, ${ }^{3}$ Sunnybrook Health Sciences Centre, ${ }^{4}$ Baycrest Health Sciences.

Background: The endocannabinoid system has been a target of interest for the treatment of agitation and is associated with AD-related changes such as brain cholesterol metabolism and neuroinflammation. We assessed whether the brain cholesterol metabolite, cerebrocholesterol (Cchol), and the proinflammatory cytokine, tumor necrosis factor (TNF) were associated with agitation severity and treatment response to the synthetic cannabinoid, nabilone.

Methods: Serum Cchol and TNF were collected from AD patients participating in a double-blind cross-over trial comparing 6 weeks of nabilone to placebo. Samples were collected at the start and end of each phase. We assessed the relationships between 1) agitation (Cohen Mansfield Agitation Inventory (CMAI) total and subscores) and each biomarker at baseline (BL); 2) changes in agitation and each biomarker at $\mathrm{BL}$; and 3) changes in agitation and changes in each biomarker.

Results: Thirty-eight patients were randomized. Cchol $(\mathrm{F}(1,36)=4.95, p=.03)$ and $\operatorname{TNF}(\mathrm{F}(2,25)=3.69, p=.04)$ were each associated with agitation at BL. In the placebo phase only, lower BL Cchol predicted increases in agitation $(\mathrm{b}=-$ $35.2, p=.02)$, and decreases in Cchol were associated with increases in agitation $(\mathrm{b}=-20.9, p=.03)$. In the nabilone phase only, lower BL TNF predicted improvements in agitation $(\mathrm{b}=-1.14, p=.045)$, and decreases in TNF were associated with improvements in agitation $(\mathrm{b}=1.12, p=.006)$.

Conclusions: Cchol and TNF may be markers of agitation severity in patients with AD. Though Cchol does not predict treatment response to nabilone, $\mathrm{Cchol}$ is associated with changes in agitation severity. Our findings with TNF suggest that this marker predicts treatment response to nabilone, and may decrease with nabilone treatment.

\section{Development of a Novel Cellular Reporter Assay to Measure Anticholinergic Activity}

Shanna C. Trenaman ${ }^{1,2}$, Melissa K. Andrew ${ }^{1,2}$, and Kerry B. Goralski ${ }^{2} .{ }^{1}$ Nova Scotia Health Authority, ${ }^{2}$ Dalhousie University.

Background: Anticholinergic activity is an important contributor to serious adverse drug events, especially in older adults. This has led to the development of numerous scales to quantify anticholinergic activity; to date these have failed to garner widespread acceptance due to their subjective nature. 
Objectives: The objective of this study was to develop a cellular reporter assay that could quantitatively measure anticholinergic activity.

Methods: The DiscoverX PathHunter ${ }^{\circledR} \beta$-Arrestin eXpress GPCR Assay was used. This assay contains human cells expressing the muscarinic 1 receptor engineered to give off light when bound by an agonist. The assay was used to identify cholinergic agonist binding and anticholinergic inhibition of agonist binding. The muscarinic agonist used was acetylcholine, and the anticholinergic agents investigated included: atropine, atenolol, famotidine, and ranitidine. Each agent was assessed across a range of concentrations. A standard luminescence plate reader (Biotek SynergyHT) was used to measure light emitted at all wavelengths. Statistical analysis was completed using GraphPad Prism 5.

Results: We used the muscarinic 1 receptor based assay to examine acetylcholine agonism and calculated the EC50 as $9778 \mathrm{nM}$. Subsequent trials to investigate inhibition of the acetylcholine induced luminescence at a concentration corresponding to the EC80 (115 $600 \mathrm{nM})$ showed complete inhibition by atropine at a concentration of $2.73 \times 10^{-5} \mathrm{M}$ and partial inhibition by varying concentrations of atenolol, famotidine, and ranitidine.

Conclusions: A cellular reporter assay can be used to measure anticholinergic activity. This objective measure of anticholinergic activity may be helpful to predict anticholinergic burden and help direct drug-therapy decisions.

\section{Computational Modeling and Simulation Methodologies in Dementia Research: Preliminary Results of a Scoping Review}

Allen McLean ${ }^{1,2,3}$, Debra Morgan ${ }^{1,2,3}$, Julie Kosteniuk ${ }^{1,2,3}$, Megan O'Connell ${ }^{1,3}$, Melanie Bayly ${ }^{1,2,3}$, Amanda Froehlich Chow $^{1,2,3}$, Valerie Elliot ${ }^{1,2,3}$, Nathaniel Osgood $^{1,3}$. ${ }^{1}$ University of Saskatchewan, ${ }^{2}$ Canadian Centre for Health and Safety in Agriculture, ${ }^{3}$ Rural Dementia in Action Research (RaDAR) CCNA Team 20.

Background: Computational science is a broad, multidisciplinary field blending advanced computing capabilities, powerful data analysis techniques, and discipline-specific knowledge for the study of complex problems that are difficult to investigate using conventional approaches.

Methods: Computational science methods are complementary to the traditional approaches of theory generation and experimentation. This rapidly evolving field includes elements of mathematical modeling (e.g. structural equation, hidden Markov, K nearest neighbours), simulation modeling (e.g. agent-based, system dynamics, discrete event), data science, complex adaptive systems theory, big data, and data analytics, and is recognized as increasingly important for the advancement of medical science. The use of these methods in human health research has increased dramatically over the past decade, and examples are found across many disciplines. As researchers and practitioners further explore the potential applications of computational science methodologies within dementia research - a timely review of the current state of knowledge is needed.

Results: This work will present preliminary results of a scoping review summarizing published research focusing on the use of computational modeling and simulation methodologies as they have been applied to questions in dementia research. We will examine the assumptions made when constructing these models, data sources and quality, the purpose of the model (e.g. predictive modeling to identify future trends and prevalence; implications for capacity planning and resource allocation; consequences for informal caregivers), and knowledge gaps that may point toward potential areas of future study.

Conclusion: The completed review will provide an up-todate and solid foundation for reference and future research on these subjects.

\section{Postdoctoral Fellows}

Consistency of Resting-State Functional MRI Connectivity Over 2.5 Years and 13 Sites Using the Canadian Dementia Imaging Protocol

AmanPreet Badhwar ${ }^{1}$, Yannik Collin-Verreault ${ }^{1}$, Pierre Orban $^{1}$, Sebastian Urchs $^{3}$, Isabelle Chouinard ${ }^{2}$, Jacob Vogel $^{3}$, Olivier Potvin ${ }^{2}$, Simon Duchesne ${ }^{2,}$, , Pierre Bellec ${ }^{1}$. ${ }^{1}$ CRIUGM, University of Montreal, ${ }^{2}$ Centre CERVO, Quebec City Mental Health Institute, ${ }^{3}$ McGill University, ${ }^{4}$ Université Laval.

Background: Resting-state functional MRI (rsfMRI) connectivity studies are increasingly acquiring data at multiple sites. An open question is how multisite acquisitions impact longitudinal connectivity measures. We assess the intra-site longitudinal consistency, as well as the intra- and inter-manufacturer consistencies of connectivity maps.

Methods: Functional images were acquired from a cognitively healthy male scanned at 13 Canadian sites on three scanner-makes, using the harmonized Canadian Dementia Imaging Protocol (CDIP). Longitudinal data (2.5 
years) was acquired at six sites. Imaging data was processed using the Neuroimage Analysis Kit. Per session, connectivity maps were extracted by computing the correlations between the time series of every voxel and the average signal in seven functional networks. Pearson's correlation was used to assess consistency between connectivity maps across visits. Consistency values from our subject were compared to values obtained from the HNU1 dataset $(\mathrm{n}=26)$.

Results: The average intra-site long-term consistency (0.66 \pm 0.11 ) across the seven networks was marginally higher than the average inter-site intra-vendor consistency $(0.62$ \pm 0.10 ). Our longitudinal finding was on par with that observed on average for each HNU1 subject (average intrasubject consistency: $0.69 \pm 0.11$ ). Average HNU1 intersubject consistency was $0.46 \pm 0.10$.

Conclusions: We demonstrate that connectivity maps with good consistency can be extracted over several years, and that the impact of acquisition sites is not large compared to the intrinsic longitudinal intrasite variations. Our results support the feasibility of standardized rsfMRI derivatives in multisite studies that extend for several years using CDIP.

\section{Neuropsychiatric Symptoms and Daily Functionality of Dementia Patients - Results from the Sunnybrook Dementia Study}

Saira Saeed Mirza ${ }^{1,2}$, Shraddha Sapkota ${ }^{2}$, Joel Ramirez ${ }^{2,3}$, Donald T. Stuss ${ }^{1}$, Nathan Herrmann ${ }^{2,5}$, Krista Lanctôt ${ }^{1,2}$, Sandra E. Black ${ }^{1,2,3^{* *}}$ and Mario Masellis ${ }^{1,2,3 * *}$. ${ }^{* *}$ Contributed equally as senior co-authors. ${ }^{1}$ University of Toronto, ${ }^{2}$ Hurvitz Brain Sciences Research Program, Sunnybrook Research Institute, ${ }^{3} \mathrm{LC}$ Campbell Cognitive Neurology Research Unit, Sunnybrook Research Institute, ${ }^{5}$ Sunnybrook Health Sciences Centre.

Background: Neuropsychiatric symptoms and functional dependence characterize all forms of dementia, such as Alzheimer's Disease (AD) and Dementia with Lewy Bodies (DLB). However, it remains unknown if neuropsychiatric symptoms predict functionality in dementia patients. In a cohort of dementia patients with AD and DLB combined, we examined if neuropsychiatric symptoms associate with or predict Activities of Daily Living (ADLs), and if this association is influenced by clinical diagnosis.

Methods: In the Sunnybrook Dementia Study, we examined if neuropsychiatric symptoms cross-sectionally associate with ADLs $(\mathrm{N}=459, \mathrm{AD}=366, \mathrm{DLB}=93)$ or longitudinally predict decline in ADLs $(\mathrm{N}=241, \mathrm{AD}=199, \mathrm{DLB}=42)$ over 1.5 years. Neuropsychiatric symptoms were assessed by the
Neuropsychiatric Inventory (NPI). Basic and Instrumental ADLs (BADL and IADL) were assessed with the Disability Assessment for Dementia. We used multiple linear regression models adjusted for age, sex, education, global cognition (Mini-Mental State Examination-MMSE), and dementia diagnosis to test associations of NPI with BADL and IADL.

Results: Mean age of the sample at baseline was $72.1 \pm 9.8$ years and $49.5 \%(n=227)$ were women. Mean age of longitudinal sample was $71 \pm 9.9$ years and $48.5 \%(n=117)$ were women. Cross-sectionally, persons with higher NPI were more likely to have worse performance on both BADL (Difference-BADL:-0.4,95\% CI:-0.5,-0.3) and IADL (Difference-IADL:-0.8,95\% CI:-0.9,-0.7) in fully adjusted models. Persons with higher NPI scores declined more over $\sim 1.5$ years on performance on BADL (Decline: $-0.2,95 \%$ CI:$0.4,-0.1$ ), but not on IADL (Decline: $0.01,95 \%$ CI:-0.2,0.2). Dementia diagnosis was not a significant confounder in any observed associations.

Conclusions: Neuropsychiatric symptoms predict increasing disability in dementia patients irrespective of dementia diagnosis. Timely treatment of neuropsychiatric symptoms may lead to some preservation of function in dementia.

\section{The Infiltration of Hematogenous Macrophages Occurs in an Age/Time and Sex-Dependent Manner in a Mouse Model of Alzheimer's Disease}

Natalie Kozyrev, Shawn Albers, Jennifer Yang, Vania F. Prado, R. Jane Rylett, Gregory A. Dekaban. Western University.

Abstract: $\beta$-Amyloid (A $\beta$ ) plaques are highly cytotoxic and trigger chronic inflammation in the cellular environment, contributing to the infiltration of macrophages (MФ) into the brain in the course of Alzheimer's disease (AD). Activated macrophages release pro-inflammatory cytokines that aggravate neurotoxicity associated with AD. A major impediment to investigating neuroinflammation involving $М \Phi$ activity is the inability to discriminate resident microglial $M \Phi$ from hematogenous $M \Phi$, as they are morphologically and phenotypically similar when activated. In order to distinguish between hematogenous and resident microglial M $\Phi$ and determine their respective roles in chronic inflammation associated with $\mathrm{AD}$ progression, we used lys-EGFP-ki transgenic mice that express enhanced green fluorescent protein (EGFP) in hematogenous $M \Phi$, but not in resident microglia. These mice were crossed with 5XFAD mice, an established aggressive model of AD. The offspring demonstrated robust $\mathrm{AD}$ pathology and enabled visual discrimination 
of hematogenous macrophages from resident microglia. Brain levels of A $\beta 1-42$ were measured with ELISA, spatial learning and memory were assessed using the Morris Water Maze and the expression of resident and hematogenous macrophages in the brain were visualized using immunofluorescence staining. Immunofluorescence data was supplemented with a quantification of total EGFP protein concentration in the cortex and hippocampus of mice using ELISA. The mice demonstrated deficits in spatial learning and robust increases in $A \beta 1-42$ by 5 months. Amyloid accumulation paralleled by the infiltration of hematogenous macrophages began earlier in female compared to male mice and preceded behavioral changes. Thus, the infiltration of hematogenous MФ occurs in an age/time dependent manner and precedes cognitive impairment.

\section{Longitudinal Analyses of Imaging Trajectories Provide Novel Insights into the Variable Dynamics of Brain Aging and Alzheimer's Disease}

G. Peggy McFall ${ }^{1}$, Olivier Potvin ${ }^{2}$, Louis Dieumengarde ${ }^{2}$, Shannon Drouin ${ }^{1}$, Richard Camicioli ${ }^{1}$, Simon Duchesne ${ }^{2}$, \& Roger A. Dixon ${ }^{1}$, for the ADNI Study. ${ }^{1}$ University of Alberta, ${ }^{2}$ Université Laval.

Background: Longitudinal models of non-pathological aging are required to achieve a better understanding of specific changes and interactions associated with AD. We have developed dynamic quantitative trajectory modeling methods with which we aim to analyze multiwave longitudinal imaging data. Specifically, we model (1) normative changes in individualized trajectory patterns, (2) precision roles of risk moderators (age, sex, APOE), and (3) subclass differences in variance, level and slope.

Method: Cognitively healthy normal adults will be drawn from ADNI data $(\mathrm{n}=432$; mean 73.8 years (range 48.1 - 91.4) at baseline; $45 \%$ females). All participants contributed magnetic resonance imaging data between one and 12 times at varying total intervals (up to eight years) that will be processed using Freesurfer 6.0 to extract cortical and subcortical measures (volumes, thicknesses, and surfaces) automatically. Longitudinal invariance testing, growth modeling, and latent class growth analyses will be performed in Mplus 7.0.

Results: First, we expect to observe significant variability in level and change for all imaging trajectory analyses, congruent with our earlier work using cross-sectional data. Second, the overall growth curves for each measure will vary when stratified by sex and APOE. Third, latent classes of trajectory patterns will vary in normative growth features and risk for impairment and AD.

Conclusions: These results will have implications for the establishment of precision-based, clinically applicable norms and future biomarker prediction analyses. Next steps will be to include individuals on the AD spectrum in multimodal dynamic prediction analyses.

\section{Understanding How Rural Primary Health Care Teams Collaborate To Deliver Dementia Care}

Amanda Froehlich Chow, Debra Morgan, Melanie Bayly, Julie Kosteniuk, Valerie Elliot. Canadian Centre for Health and Safety in Agriculture, University of Saskatchewan.

Background: Individuals living in rural areas have limited access to dementia-specific primary health care (PHC) services. Moreover, little is known about the processes for interdisciplinary team-based care, particularly in rural settings. In an effort to address these gaps, the Rural Dementia Action Research (RaDAR) Team is developing evidence-based best practices for delivering PHC to rural residents living with dementia.

Aim: The primary focus of this study is to understand how rural PHC teams deliver care to those living with dementia. Collaborative processes among PHC teams caring for both the general population, and older adults with chronic conditions, will also be explored.

Research Questions: 1) What are the goals of rural PHC teams who are working toward engaging in collaborative care approaches? 2) What specific strategies do rural PHC teams apply when working collaboratively? 3) What are the experiences of rural PHC team members when collaborating to deliver care? 4) What are the key challenges that rural PHC teams face; and (if applicable) how have teams overcome these challenges?

Methods: A community-based research approach, employing a case study design will guide this study. Participants will include 4 PHC teams located in rural Saskatchewan (2 of the 4 teams will be enrolled in the RaDAR study). Data will be collected via one-on-one and focus group interviews, surveys, direct observation and document review.

Impact: Team-based collaborative processes identified in this project will be evaluated and incorporated (where applicable); in turn, supporting the RaDAR team's broader goals of developing evidence-based best practices for dementia-specific integrated rural PHC. 
A Scoping Review of Dementia-Related Education and Support Service Accessibility and Use in Rural Areas: Barriers and Promising Solutions

Melanie Bayly, Debra Morgan, Amanda Froehlich Chow, Julie Kosteniuk, Valerie Elliot. University of Saskatchewan.

Objectives: The Rural Dementia Action Research (RaDAR) team is working to improve the quality of life and care for individuals affected by dementia in rural areas. In line with this work, the current research aimed to map and synthesize the published literature related to education and support services for individuals with dementia and their caregivers living rurally. The objectives of this review were to: 1) Identify the services available to these populations and any gaps/service needs; and 2) Identify barriers to service access and use, and promising solutions to these barriers.

Methods: Empirical, English language articles (2381) from the past 20 years were identified within MEDLINE, CINAHL, PSYCINFO, and EMBASE using terms related to dementia, rural, support services, and education. Articles were screened according to Arksey and O'Malley's (2005) five-stage scoping review methodology, and 68 articles were included after full-text review.

Results: Findings suggest limited availability of dementiarelated support and education services in most rural contexts, particularly respite care and day programs. Service use varied across studies, with barriers including low knowledge of services, practicality and resource issues (e.g., transportation, financial), values and beliefs, stigma, and negative perceptions of services. Solutions included tailored and person-centered services, technological service provision, accessibility considerations and assistance, inter-organization collaboration, education on services, technological service provision, and having a "point of entry" to service use.

Implications: Findings illustrate both challenges and promising innovations in rural dementia education and support services, and can inform the development of accessible services that are used by rural populations.

Regional Brain Glucose Uptake Is Unrelated to Tau and AB Deposition in the 3xTg Mouse Model of Alzheimer's Disease

Aida Adlimoghaddam ${ }^{1,2 *}$, Claudia Perez ${ }^{1}$, Greg Stortz ${ }^{3}$, Jelena Djordjevic ${ }^{1}$, Wanda M. Snow ${ }^{1}$, Andrew LGoertzen ${ }^{2}$, Ji Hyun $\mathrm{Ko}^{2}$, Benedict C Albensi ${ }^{1,2^{*}}$. ${ }^{1}$ St. Boniface Hospital Research, ${ }^{2}$ University of Manitoba, ${ }^{3}$ University of British Columbia.
Background: Alzheimer's disease (AD) is a progressive agerelated neurodegenerative disease. Although neurofibrillary tangles (NFTs) and amyloid beta (A $\beta$ ) are common hallmarks of $\mathrm{AD}$, the earliest deficits in the pathological progression of $\mathrm{AD}$ seem to be caused by impaired brain metabolism. Therefore, efforts to detect alterations in brain metabolism could improve our ability to diagnose AD in early stages and identify more effective treatment targets.

Methods: The current study utilized FDG-PET to measure abnormalities in brain metabolic activity in AD mice and control mice. Both 3xTg-AD mice and control mice underwent FDG-PET neuroimaging. Further, congo red staining and immunohistochemistry were utilized to detect neuropathology of $A \beta$ and NFTs. Moreover, we measured the activity of mitochondrial enzymes involved in the metabolic pathways. Western blotting was used to determine protein levels of Complex I-V subunits.

Results: We found significant brain hypometabolic changes $(p<0.01)$ as measured by FDG-PET in cortical insular and piriform regions of $\mathrm{AD}$ brains compared to that of control brains. Also, we found a significant reduction $(p<0.05)$ in the activity of mitochondrial cytochrome c oxidase as well as significant decreases $(p<0.05)$ in expression of mitochondrial complex protein subunits in AD brain as compared to control brains. No significant differences were found in the activity of citrate synthase or GAPDH between AD and controls. No congophilic positive $A \beta$ was detected in any brain region in either $\mathrm{AD} /$ control mice; however, we detected intracellular $A \beta$ in the entorhinal cortex and NFTs in the entorhinal cortex, parasubiculum, and sagulum of AD mice.

Conclusions: No association was found between neuropathological and functional changes in AD sensitive brain regions, suggesting that brain hypometabolism occurs prior to $\mathrm{AD}$ pathology. Therefore, targeting metabolic mechanisms in cortical piriform and insular regions emerges as a promising interventional target for preventing, slowing, and/or blocking the onset of AD.

\section{CCNA Investigator Abstracts}

CCNA Team 20 Rural: A Scoping Review of Multiple Transitions Across Care Settings Among Individuals with Dementia

Julie Kosteniuk $^{1}$, Debra Morgan ${ }^{1}$, Valerie Elliot ${ }^{1}$, Amanda Froehlich Chow ${ }^{1}$, Melanie Bayly ${ }^{1}$, Erin Watson ${ }^{1}$, Meric Osman $^{2}$, Beliz Acan Osman ${ }^{2}$, Megan E. O'Connell', Andrew Kirk ${ }^{1}$, Norma Stewart ${ }^{1}$, Allison Cammer ${ }^{1}$, Anthea Innes ${ }^{3} .{ }^{1}$ University of Saskatchewan, ${ }^{2}$ Saskatchewan Health Quality Council, ${ }^{3}$ University of Salford. 
Background: Multiple transitions across care settings can be disruptive for older adults with dementia and result in fragmented care.

Objective: To identify patterns of multiple transitions experienced by individuals with dementia, including number of transitions and care settings involved, and the factors associated with multiple transitions.

Methods: The review followed the Arksey and O'Malley five-stage scoping review methodology and further steps proposed by Levac et al. Peer-reviewed studies published between 2007 and 2017 were identified in searches of three databases (Medline, EMBASE, and CINAHL). Multiple transitions were defined as either two or more moves between at least three different care settings (e.g., homehospital-long term care), or three or more moves between at least two different care settings (e.g., home-hospitalhome-hospital).

Results: Thirty-three articles were included in the review. A total of 26 multiple transition routes were identified based on the care settings involved and number of transitions. The most frequent two-transition route was characterized by admission from home to hospital followed by admission to long-term care. Single transitions from hospital to long-term care were associated with sex, older age, marital status, and health characteristics such as comorbidity and functioning. Hospital admission and readmission from unspecified settings defined most of the routes that consisted of more than two transitions.

Implications: There remain gaps in understanding movement patterns across care sites and duration of stays between transitions. To provide support and prevent avoidable transitions, greater attention should also be given to particularly vulnerable sub-populations undergoing transitions (e.g., ethnic groups, rural communities).

\section{Rationale and Design of the Trial of Remote Ischemic Conditioning for Vascular Cognitive Impairment (TRIC-VCI)}

Eric E. Smith ${ }^{1}$, Philip A. Barber ${ }^{2}$, Thalia S. Field ${ }^{3}$, Richard Frayne $^{2}$, Aravind Ganesh ${ }^{4}$, Vladimir Hachinski ${ }^{5}$, Cheryl R. McCreary ${ }^{1}$, Leonardo Pantoni ${ }^{6}$, Demetrios J. Sahlas ${ }^{7}$, Mukul Sharma ${ }^{7}$, Richard H. Swartz ${ }^{8}$, Dale Corbett ${ }^{9}$, Sandra E. Black ${ }^{10}$. ${ }^{1}$ University of Calgary, ${ }^{2}$ Hotchkiss Brain Institute, ${ }^{3}$ University of British Columbia, ${ }^{4}$ University of Oxford, ${ }^{5}$ Western University, ${ }^{6}$ University of Florence, ${ }^{7}$ McMaster University, ${ }^{8}$ Sunnybrook Health Sciences Centre, ${ }^{9}$ University of Ottawa, ${ }^{10}$ Sunnybrook Research Institute.
Introduction: Patients with cerebral small vessel disease are at risk for ischemia and vascular cognitive impairment (VCI). Experimentally, inducing brief periods of ischemiareperfusion in a limb (i.e. remote ischemic conditioning, RIC) reduces subsequent ischemic injury in the brain.

Methods: Inclusion criteria include age 60-85, cerebral small vessel disease on brain CT or MRI with either confluent or beginning confluent white matter lesions or multiple lacunar infarcts, MoCA 13-24, and preserved basic activities of living. Twenty-four participants will be randomized to one of two RIC intensity arms: one arm treated once per day or one arm treated twice per day for 30 consecutive days. RIC will consistent of 4 cycles of 5 minutes of blood pressure cuff inflation to systolic + $20 \mathrm{mmHg}$, alternating with 5 minutes deflation (total 35 minutes).

Results: Outcomes will be assessed at 30 days and 90 days (i.e. 60 days after ceasing treatment). The primary outcome is adherence, defined as completing $\geq 80 \%$ of the sessions. Secondary outcomes include the percent of sessions completed and participant-reported pain/ discomfort. Efficacy outcomes will include cerebral blood flow measured by ASL MRI, white matter hyperintensity progression, white matter DTI changes, and change in MoCA and Trail-Making tests. In parallel with the human RCT, animal experiments will explore effects of varying RIC intensity, duration and timing.

Conclusions: This translational interventional research program will provide information on feasibility, dose, adherence, tolerability and surrogate outcome markers that will be used to design a phase $2 \mathrm{~b}$ randomized controlled trial of RIC in CCNA Phase 2.

\section{Perspectives from the Field: Designing the Driving Cessation in Dementia Intervention Toolkit (DCD-IT)}

Gary Naglie ${ }^{1,2}$, Stéphanie Yamin ${ }^{3}$, Brenda Vrkljan ${ }^{4}$, Holly Tuokko ${ }^{5}$, Sarah Sanford ${ }^{1}$, Elaine Stasiulis ${ }^{1}$, Michelle Porter ${ }^{6}$, Jan Polgar ${ }^{7}$, Anita Myers ${ }^{8}$, Paige Moorhouse ${ }^{9}$, Frank Molnar $^{10}$, Barbara Mazer ${ }^{11}$, Shawn Marshall ${ }^{10}$, Isabelle Gélinas $^{11}$, Alexandra Crizzle ${ }^{12}$, Anna Byszewski ${ }^{10}$, Patricia Belchior ${ }^{11}$, Michel Bédard ${ }^{13}$, and Mark Rapoport ${ }^{2}$ on behalf of the CCNA Driving Cessation Team Co-Investigators and CCNA study group. ${ }^{1}$ Baycrest Health Sciences, 2University of Toronto, ${ }^{3}$ St. Paul University, ${ }^{4}$ McMaster University, ${ }^{5}$ University of Victoria, ${ }^{6}$ University of Manitoba, ${ }^{7}$ Western University, ${ }^{8}$ University of Waterloo, ${ }^{9}$ Dalhousie University, ${ }^{10}$ University of Ottawa, ${ }^{11} \mathrm{McGill}$ University, ${ }^{12}$ University of Saskatchewan, ${ }^{13}$ Lakehead University, ${ }^{14}$ Sunnybrook Health Sciences. 
Background: Decision-making about driving cessation and transitioning to non-driving is a challenging and complex issue faced by drivers diagnosed with dementia and their families. To address the gap in evidence-based interventions that support individuals in the driving cessation process, we developed the Driving Cessation in Dementia Intervention Toolkit (DCD-IT).

Objectives: To further develop and refine the DCD-IT (content, design and mode of delivery) to facilitate its effective implementation in settings that support older adults with dementia.

Methods: Representatives ( $\mathrm{n}=15)$ from Alzheimer Society organizations in British Columbia, Manitoba, Ontario and Nova Scotia reviewed the DCD-IT and provided feedback via a webinar, questionnaire and in-depth interviews. Data analysis techniques included thematic coding and inductive analysis.

Results: Participants emphasized the lack of accessible and trusted driving cessation resources specific to meeting the needs of PWD and their family caregivers. Gaps and corresponding areas of focus they identified to inform the continued development and design of the DCD-IT included: materials directed separately to PWD encompassing appropriate content, language and design; resources geared to helping healthcare providers engage in discussions with PWD and family caregivers; information for PWD, family caregivers and healthcare providers about region-specific driving regulations and alternative transportation options.

Implications: Obtaining the perspective of AS representatives have informed improvements to the content and design of the DCD-IT. Next steps include evaluating the implementation process of the intervention framework and toolkit in AS chapters in community settings in Ontario with the aim of informing widespread implementation and adaption across Canada.

\section{Digital Storytelling in Persons with Dementia: Findings from a Multi-Site Study}

Lili Liu ${ }^{1}$, Kara Hollinda ${ }^{1}$, David Kaufman ${ }^{3}$, Arlene Astell ${ }^{2}$, Christine Daum ${ }^{1}$. ${ }^{1}$ University of Alberta, ${ }^{2}$ University of Toronto, ${ }^{3}$ Simon Fraser University.

Background: Digital stories combine voices, images or video, and music to describe events in people's lives. Digital stories can help people with cognitive impairment to reminisce and communicate with others. The creation of digital stories in persons with early to moderate dementia can also support self-expression and identity.
Purposes: 1) Determine the feasibility of co-creating digital stories by persons in early to moderate stages of dementia; 2) Describe the types of content chosen by the participants; 3) Describe facilitators' roles in digital storytelling.

Methods: Participants with dementia were recruited in Vancouver $(n=6)$, Edmonton $(n=7)$, and Toronto $(n=7)$. Participants ranged in age from 63 to 91 years. In sessions over 8-10 weeks, a facilitator (researcher) co-created stories with participants using WeVideo software. Sessions were audiorecorded and subjected to qualitative content analysis. Digital stories were shown to family caregivers in each city.

Findings: Participants with dementia were able to cocreate digital stories. Digital stories were 3 to 7 minutes long and focused on family, personal accounts of war, travel, and sports. Content related to storytellers' roles that represented their identities. Participants with moderate dementia preferred shorter, more frequent sessions than those with mild dementia. Preliminary analyses of facilitator roles revealed that key communication strategies were active listening, strategic questioning, comfort with silence, and therapeutic responding. Building relationships and collaboration were achieved through flexibility, empathy, and enablement of autonomy, competence, and meaning. Facilitators were keen observers, flexible, and adapted specific communication strategies and relational skills to the strengths and "in-the-moment" needs of the participants.

Team 20 Rural: Development, Implementation, and Scaling up of a Rural Primary Health Care Model for Dementia

Debra Morgan $^{1}$, Julie Kosteniuk ${ }^{1}$, Megan E. O'Connell', Andrew Kirk ${ }^{1}$, Norma J. Stewart ${ }^{1}$, Dallas Seitz ${ }^{2}$, Jayna Holroyd-Leduc ${ }^{3}$, Jean Daku ${ }^{4}$, Tracy Hack ${ }^{4}$, Faye Hoium ${ }^{4}$, Deb Kennett-Russill ${ }^{4}$, Kristen Sauter ${ }^{4}$. ${ }^{1}$ University of Saskatchewan, ${ }^{2}$ Queen's University, ${ }^{3}$ University of Calgary, ${ }^{4}$ Saskatchewan Health Authority (Sun Country).

Background: Limited specialist and service access in rural areas leads to increased responsibility for dementia diagnosis and management in primary health care (PHC). However, a gap exists in evidence-based best practices for dementia care in rural PHC settings.

Aim: Using a community-based approach, the focus of Team 20 Rural in CCNA Phase I was to collaborate with rural PHC providers to develop a sustainable and scalable model of PHC that consists of integrated clinical support tools and education. 
Methods: Elements of the Rural PHC Model for Dementia are based on seven principles of effective PHC for dementia identified from research, organized into three domains: interprofessional care, decision support, and specialistto-provider support. Using a 5-step adaptation approach, we collaborated with a rural PHC team to operationalize these elements for their practice in a small Saskatchewan community (pop. 1,000).

Results: Interprofessional care was integrated into a decision support tool and family-centred case conferences were introduced. The decision support tool is based on the Primary Care-Dementia Assessment \& Treatment Algorithm (PC-DATA ${ }^{\mathrm{TM}}$ ) and flow sheets have been added to the electronic medical record. Specialist-to-provider education sessions on topics identified by the PHC team are provided by urban-based specialists with Team 20 Rural (neurologist and neuropsychologist).

Implications: We are adapting this model with two additional PHC teams in rural communities of 1,000 and 10,000 population. In CCNA Phase II, we will implement and evaluate a novel model of remote healthcare where a highly-trained urban-based clinician delivers a suite of interventions to rural/remote Saskatchewan communities via telehealth.

\section{Examining Changes in Trends in Health System Performance for Men and Women Diagnosed with Dementia: A Population-Based Study in Ontario}

Nadia Sourial ${ }^{1,2}$, Susan Bronskill ${ }^{2,3}$, Claire GodardSebillotte $^{1}$, Jacob Etches $^{2}$, Isabelle Vedel ${ }^{1} .{ }^{1}$ McGill University, ${ }^{2}$ Institute for Clinical Evaluative Sciences, ${ }^{3}$ Institute of Health Policy Management \& Evaluation, University of Toronto.

Objective: To examine how trends in health system performance have changed following the introduction of enhanced primary care models in Ontario for men and women with dementia.

Methods: Population-based, repeated, cohort study of community-dwelling adults $65+$ years in Ontario, newly diagnosed with dementia in each year between 2002 and 2014 and followed for one year. Thirty indicators of health system performance were derived from the Health Quality Ontario framework and the Canadian consensus guidelines in dementia. Variations in indicators over time were represented graphically, stratified by sex.

Results: The number of incident cases in men increased from 7,773 in 2002 to 10,070 in 2014; cases in women increased from 12,096 to 14,172 . Visits to primary care $(\sim 11$ per year) and specialists $(\sim 2$ per year) were similar and stable over time in both men and women. Home care visits doubled from 13 to 26 visits in women and from 10 to 20 visits per year in men. Long-term care admissions decreased by $5 \%$ in both sexes. The rate of avoidable hospitalizations decreased slightly by $1-2 \%$ over time in both sexes. Results on other indicators will also be presented.

Implications: This study is among the first to track health system performance and to contrast sex differences in newly-diagnosed persons in Ontario. Few sex differences over time were observed. While some indicators improved overall, the relationship between the introduction of new primary care models and these changes needs to be further explored.

\section{Factors Associated with Cognitive Decline in a Home Care Population with Existing Cognitive Challenges: Survival Analysis Using RAI-HC Data for Ontario}

Dawn M. Guthrie ${ }^{1}$, Nicole Williams ${ }^{1}$, Jennifer Campos ${ }^{2}$, Paul Mick ${ }^{3}$, Joseph B. Orange ${ }^{4}$, M. Kathleen PichoraFuller $^{5}$, Natalie A. Phillips 6 , Marie Savundranayagam ${ }^{4}$, Walter Wittich ${ }^{7}{ }^{1}$ Wilfrid Laurier University, ${ }^{2}$ Toronto Rehabilitation Institute, ${ }^{3}$ University of British Columbia at Kelowna, ${ }^{4}$ Western University, ${ }^{5}$ University of Toronto at Mississauga, ${ }^{6}$ Concordia University, ${ }^{7}$ Université de Montréal.

Background: Older adults are likely to experience impairments in sensory and cognitive functioning and few studies have examined how sensory losses influence deterioration in cognitive abilities.

Methods: Secondary analysis of existing electronic records from the Resident Assessment Instrument for Home Care explored the influences of both vision and hearing impairment, alone and in combination (dual sensory impairment) in a sample of Canadian home care clients with cognitive impairment at baseline $(\mathrm{n}=30,623)$. Time to cognitive decline, measured using the Cognitive Performance Scale (CPS), was analyzed using Cox proportional hazards models.

Results: Clients with a baseline CPS score of four had the fastest mean time to decline at 21.8 months and this was true among all three sensory impairment groups. After multivariate adjustment, the risk of cognitive decline was increased among clients who were male $(\mathrm{HR}=1.14$; CI: $1.10,1.19)$, had vision impairment (HR $=1.08$; CI: $1.01,1.16)$ or a dual sensory impairment $(\mathrm{HR}=1.10 ; \mathrm{CI}: 1.03,1.17)$. 
Conclusions: Older home care clients who are experiencing mild cognitive impairment are likely to experience further declines in cognitive functioning and this is particularly true in the presence of a dual sensory loss. These individuals represent an important group that should be assessed and monitored closely in order to flag early declines in capacity so that proper interventions can be put in place to try and delay further losses.

Measuring the Fidelity of a Policy Implementation: Insights from the Quebec Alzheimer Plan

Marine Hardouin ${ }^{1}$, Genevieve Arsenault-Lapierre ${ }^{1,2}$, Claire Godard-Sebillotte $^{2}$, Nadia Sourial ${ }^{1,2}$, Howard Bergman ${ }^{2}$, Isabelle Vedel ${ }^{1,2}$. ${ }^{1}$ Lady Davis Institute, Jewish General Hospital, ${ }^{2} \mathrm{McGill}$ University.

Context: In 2014, the Quebec Alzheimer Plan (AP) was launched in 38 family medicine groups (FMGs) to improve the ability of primary care professionals to detect and care for patients with Alzheimer's disease and their caregivers. Objective: Describe the fidelity of implementation of the AP in participating FMGs

Method: 42-item questionnaire measuring the fidelity of the AP implementation, distributed via mail to the medical directors in 38 FMGs in 2016/2017. Face-validity and content validity were achieved through expert opinion, a "Think Aloud" process and a clinical sensitivity survey. Main and Secondary Outcome

Measures: Overall degree of fidelity of the AP implementation, and subscales' scores. Scores of less than $6 / 10$ were considered low fidelity.

Results: 27 out of 38 questionnaires (71\%) have been returned. FMGs reported an overall degree of fidelity of 5.6/10 (SD 1.2). They also reported the following scores in the subscales: 5.3/10 (2.2) on Organizational Health Care System Delivery; 5.3/10 (1.1) on Decision Support; 6.9/10 (2.1) on Clinical Information System; 6.3/10 (1.5) on Delivery System Design; 6.1/10 (2.2) on Self-management Support; 6.0/10 (1.1) on Community Linkage; 4.4/10 (2.4) on Integration of Chronic Care Model Components; and 6.0/10 (1.8) on Innovation.

Conclusions: Two years after the launch of the Alzheimer Plan, its implementation is not complete: in particular in clinical leadership and financial support, decision support tools, community linkage and coordination of care. Measuring fidelity of a policy implementation with a reliable tool is the first mandatory step for a sound impact evaluation.
Family Physicians' Attitude, Knowledge and Practice Regarding Dementia Care and Dementia Initiatives in Quebec, Ontario and New Brunswick

Genevieve Arsenault-Lapierre ${ }^{1}$, Marine Hardouin ${ }^{1}$, Nadia Sourial ${ }^{1,2}$, Howard Bergman ${ }^{1}$, Isabelle Vedel ${ }^{1,2}{ }^{1}$ Lady Davis Institute, Jewish General Hospital, ${ }^{2} \mathrm{McGill}$ University.

Context: Many provinces are implementing initiatives to better prepare primary care to tackle dementia care. In Quebec, a provincial plan was implemented in 2014. In Ontario, many local initiatives have been put in place since 1999 and similar initiatives are under consideration in New Brunswick. Knowledge, attitudes, and practices of the family physicians might be key factors in the uptake of such initiatives. The goal of this study is to measure the knowledge, attitudes, and practices regarding dementia care of the family physicians involved in these initiatives from the three provinces.

Methods: We distributed a validated 72-item questionnaire to 1059 physicians in Quebec, Ontario and New Brunswick between 2014 and 2018. Descriptive analyses will be performed on five subdomains: 1) perceived competency and knowledge, 2) attitudes toward the dementia initiatives, 3) practices for cognitive evaluation, 4) attitudes toward dementia care, and 5) attitudes toward collaboration with nurses and other professionals.

Results/Conclusion: Descriptive results for each of these subdomains will be presented. These results will inform our next step in identifying the key factors associated with best practices in dementia care.

\section{Lipidomics Analyses in Parkinson's Disease}

Richard Camicioli, Roger A. Dixon, Jaspaul Tadley, Adriana Zardini Buzatto, Dorothea Mung, Barinder Bajwa, Liang Li. University of Alberta.

Background: Parkinson's disease (PD) is the most common neurodegenerative movement disorder. There are no effective biomarkers for diagnosing or tracking the disease progression. Metabolomics studies have shown promise and lipid studies suggest distinct profiles in PD. We compare the lipidomes of 43 healthy control samples and 43 PD samples with two subgroups: PD with no dementia (PDND) and PD with incipient dementia (PDID).

Methods: An untargeted lipidomics profiling method applied ultra-high pressure liquid chromatography 
(UHPLC) combined with ultra-high resolution (UHR) quadrupole time-of-flight mass spectrometry (QTOF-MS) to serum samples obtained at baseline. Bruker Profile Analysis software was used to process the LC-MS data. MetaboAnalyst was used for statistical analysis.

Results: Over 4391 lipid features were consistently detected in more than $50 \%$ of the samples. Among them, 406 lipids could be identified, spanning 12 lipid classes. We determined five lipids for distinguishing the overall PD and control groups. In receiver operating characteristic (ROC) analysis, these putative biomarkers gave an area-under-the-curve
(AUC) of 0.976 with high sensitivity (92\%) and specificity (90\%). Within the PD group, the same five-lipid panel plus another compound discriminated PDID from PDND (AUC $=0.958$ ) with sensitivity of $87 \%$ and specificity of $94 \%$. We also observed an increasing trend of the 5 common lipids in concentration, suggesting the potential of using these biomarkers for not only diagnosis of PD, but also tracking PD progression into PDID.

Conclusion: Serum lipidomics analyses produce promising prognostic markers in $\mathrm{PD}$ that may also provide insights into mechanisms of cognitive decline in PD. 